International Journal of Pure and Applied Mathematics

Volume 93 No. 1 2014, 95-107

ISSN: 1311-8080 (printed version); ISSN: 1314-3395 (on-line version)

url: http://www.ijpam.eu

doi: http://dx.doi.org/10.12732/ijpam.v93i1.8

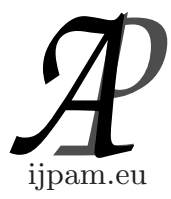

\title{
L-JOIN MEET APPROXIMATION OPERATORS
}

\author{
Yong Chan Kim \\ Department of Mathematics \\ Gangneung-Wonju University \\ Gangneung, Gangwondo 210-702, KOREA
}

\begin{abstract}
In this paper, we define $L$-join meet and $L$-meet join approximation operators as a generalization of fuzzy rough set in complete residuated lattices. We show that $L$-join meet and $L$-meet join approximation operators are induced by various $L$-fuzzy relations. We investigate relations between their operations and Alexandrov $L$-topologies.
\end{abstract}

AMS Subject Classification: 03E72, 03G10, 06A15, 06F07

Key Words: complete residuated lattices, $L$-join meet and $L$-meet join approximation operators, Alexandrov $L$-topologies

\section{Introduction}

Pawlak $[7,8]$ introduced rough set theory as a formal tool to deal with imprecision and uncertainty in data analysis. Hájek [2] introduced a complete residuated lattice which is an algebraic structure for many valued logic. Radzikowska [9] developed fuzzy rough sets induced by various $L$-fuzzy relations in complete residuated lattice. Bělohlávek [1] investigated information systems and decision rules in complete residuated lattices. Zhang [5,6] introduced Alexandrov $L$-topologies induced by fuzzy rough sets. Algebraic structures of fuzzy rough sets are developed in many directions $[3,4,10,11]$

In this paper, we introduce $L$-join meet and $L$-meet join approximation operators as a generalization of fuzzy rough set in complete residuated lattices.

Received: November 22, 2013

(c) 2014 Academic Publications, Ltd. url: www.acadpubl.eu 
We show that $L$-join meet and $L$-meet join approximation operators are induced by various $L$-fuzzy relations. We investigate relations between their operations and Alexandrov $L$-topologies.

\section{Preliminaries}

Definition 1. [1,2] An algebra $(L, \wedge, \vee, \odot, \rightarrow, \perp, \top)$ is called a complete residuated lattice if it satisfies the following conditions:

(C1) $L=(L, \leq, \vee, \wedge, \perp, \top)$ is a complete lattice with the greatest element $\top$ and the least element $\perp$;

(C2) $(L, \odot, \top)$ is a commutative monoid;

(C3) $x \odot y \leq z$ iff $x \leq y \rightarrow z$ for $x, y, z \in L$.

In this paper, we assume $\left(L, \wedge, \vee, \odot, \rightarrow,^{*} \perp, \top\right)$ is a complete residuated lattice with the law of double negation;i.e. $x^{* *}=x$. For $\alpha \in L, A, \top_{x} \in L^{X}$, $(\alpha \rightarrow A)(x)=\alpha \rightarrow A(x), \quad(\alpha \odot A)(x)=\alpha \odot A(x)$ and $\top_{x}(x)=\top, \top_{x}(y)=$ $\perp$, otherwise.

Lemma 2. $[1,2]$ For each $x, y, z, x_{i}, y_{i} \in L$, we have the following properties.

(1) If $y \leq z, x \odot y \leq x \odot z, x \rightarrow y \leq x \rightarrow z$ and $z \rightarrow x \leq y \rightarrow x$.

(2) $x \rightarrow\left(\bigwedge_{i \in \Gamma} y_{i}\right)=\bigwedge_{i \in \Gamma}\left(x \rightarrow y_{i}\right)$.

(3) $\left(\bigvee_{i \in \Gamma} x_{i}\right) \rightarrow y=\bigwedge_{i \in \Gamma}\left(x_{i} \rightarrow y\right)$.

(4) $\bigwedge_{i \in \Gamma} y_{i}^{*}=\left(\bigvee_{i \in \Gamma} y_{i}\right)^{*}$ and $\bigvee_{i \in \Gamma} y_{i}^{*}=\left(\bigwedge_{i \in \Gamma} y_{i}\right)^{*}$.

(5) $(x \odot y) \rightarrow z=x \rightarrow(y \rightarrow z)=y \rightarrow(x \rightarrow z)$.

(6) $x \odot y=\left(x \rightarrow y^{*}\right)^{*}$.

(7) $x \odot(x \rightarrow y) \leq y$.

(8) $(x \rightarrow y) \odot(y \rightarrow z) \leq x \rightarrow z$.

Definition 3. [1,5] Let $X$ be a set. A function $R: X \times X \rightarrow L$ is called:

(R1) reflexive if $R(x, x)=\top$ for all $x \in X$,

(R2) symmetric if $R(x, x)=\top$ for all $x \in X$,

(R3) transitive if $R(x, y) \odot R(y, z) \leq R(x, z)$, for all $x, y, z \in X$.

(R4) Euclidean if $R(x, z) \odot R(y, z) \leq R(x, y)$, for all $x, y, z \in X$.

If $R$ satisfies (R1) and (R3), $R$ is called an $L$-fuzzy preorder.

If $R$ satisfies (R1), (R2) and (R3), $R$ is called an $L$-fuzzy equivalence relation. 


\section{L-Join Meet Approximation Operators}

Definition 4. A map $\mathcal{K}: L^{X} \rightarrow L^{X}$ is called an $L$-join meet approximation operator iff it satisfies the following conditions

$(\mathrm{K} 1) \mathcal{K}(A) \leq A^{*}$

(K2) $\mathcal{K}(\alpha \odot A)=\alpha \rightarrow \mathcal{K}(A)$,

(K3) $\mathcal{K}\left(\bigvee_{i \in I} A_{i}\right)=\bigwedge_{i \in I} \mathcal{K}\left(A_{i}\right)$

A map $\mathcal{M}: L^{X} \rightarrow L^{X}$ is called an $L$-meet join approximation operator iff it satisfies the following conditions

(M1) $A^{*} \leq \mathcal{M}(A)$,

(M2) $\mathcal{M}(\alpha \rightarrow A)=\alpha \odot \mathcal{M}(A)$,

(M3) $\mathcal{M}\left(\bigwedge_{i \in I} A_{i}\right)=\bigvee_{i \in I} \mathcal{M}\left(A_{i}\right)$.

Lemma 5. (1) If $\mathcal{K}$ is an $L$-join meet approximation operator, then $\alpha \odot$ $\mathcal{K}(A) \leq \mathcal{K}(\alpha \rightarrow A)$.

(2) If $\mathcal{M}$ is an $L$-meet join approximation operator, then $\mathcal{M}(\alpha \odot A) \leq \alpha \rightarrow$ $\mathcal{M}(A)$.

Proof. (1) If $A \leq B$, by (K3), $\mathcal{K}(B)=\mathcal{K}(A \vee B)=\mathcal{K}(A) \wedge \mathcal{K}(B)$, that is, $\mathcal{K}(B) \leq \mathcal{K}(A)$. Since $\alpha \odot(\alpha \rightarrow A) \leq A$ from Lemma $2(7)$,

$$
\mathcal{K}(A) \leq \mathcal{K}(\alpha \odot(\alpha \rightarrow A))=\alpha \rightarrow \mathcal{K}(\alpha \rightarrow A) .
$$

Hence $\alpha \odot \mathcal{K}(A) \leq \mathcal{K}(\alpha \rightarrow A)$.

(2) Since $\mathcal{M}(A) \leq \mathcal{M}(B)$ for $B \leq A$ from (M3), for $A \leq \alpha \rightarrow \alpha \odot A$, we have

$$
\mathcal{M}(\alpha \rightarrow \alpha \odot A)=\alpha \odot \mathcal{M}(\alpha \odot A) \leq \mathcal{M}(A) .
$$

Hence $\mathcal{M}(\alpha \odot A) \leq \alpha \rightarrow \mathcal{M}(A)$.

Theorem 6. (1) A map $\mathcal{K}: L^{X} \rightarrow L^{X}$ is an $L$-join meet approximation operator iff there exists a reflexive $L$-fuzzy relation $R^{*} \in L^{X \times X}$ such that

$$
\mathcal{K}(A)(y)=\bigwedge_{x \in X}(A(x) \rightarrow R(x, y)) .
$$

(2) A map $\mathcal{K}: L^{X} \rightarrow L^{X}$ is an $L$-join meet approximation operator with $\mathcal{K}\left(\mathcal{K}^{*}(A)\right)=\mathcal{K}(A)$ iff there exists an $L$-fuzzy preorder $R^{*} \in L^{X \times X}$ such that

$$
\mathcal{K}(A)(y)=\bigwedge_{x \in X}(A(x) \rightarrow R(x, y)) .
$$


(3) A map $\mathcal{K}: L^{X} \rightarrow L^{X}$ is an $L$-join meet approximation operator with $\mathcal{K}(\mathcal{K}(A))=\mathcal{K}^{*}(A)$ iff there exists a reflexive and Euclidean $L$-fuzzy relation $R^{*} \in L^{X \times X}$ such that

$$
\mathcal{K}(A)(y)=\bigwedge_{x \in X}(A(x) \rightarrow R(x, y)) .
$$

Proof. $(1)(\Rightarrow)$ Define $R(x, y)=\mathcal{K}\left(\top_{x}\right)(y)$. By $(\mathrm{K} 1), R(x, x)=\mathcal{K}\left(\top_{x}\right)(x) \leq$ $\mathrm{T}_{x}^{*}(x)$. So, $R(x, x)=\perp$. Since $A=\bigvee_{x \in X}\left(A(x) \odot \mathrm{\top}_{x}\right)$, we have

$$
\begin{aligned}
\mathcal{K}(A)(y) & =\mathcal{K}\left(\bigvee_{x \in X}\left(A(x) \odot \top_{x}\right)\right)(y) \\
& =\bigwedge_{x \in X}\left(A(x) \rightarrow \mathcal{K}\left(\top_{x}\right)(y)\right) \\
& =\bigwedge_{x \in X}(A(x) \rightarrow R(x, y)) .
\end{aligned}
$$

$(\Leftarrow)(\mathrm{K} 1) \mathcal{K}(A)(y)=\bigwedge_{x \in X}(A(x) \rightarrow R(x, y)) \leq A(y) \rightarrow R(y, y)=A^{*}(y)$.

$(\mathrm{K} 2) \mathcal{K}(\alpha \odot A)(y)=\bigwedge_{x \in X}(\alpha \odot A(x) \rightarrow R(x, y))=\alpha \rightarrow \bigwedge_{x \in X}(A(x) \rightarrow$ $R(x, y))=\alpha \rightarrow \mathcal{K}(A)(y)$.

(2) $(\Rightarrow)$ By $(1)$, we define $R(x, y)=\mathcal{K}\left(\top_{x}\right)(y)$. Since $\mathcal{K}\left(\mathcal{K}^{*}\left(\top_{x}\right)\right)(z) \geq$ $\mathcal{K}\left(\top_{x}\right)(z)$ and $\mathcal{K}^{*}\left(\top_{x}\right)=\bigvee_{y \in X}\left(\mathcal{K}^{*}\left(\top_{x}\right)(y) \odot \top_{y}\right.$, we have

$$
\begin{aligned}
\mathcal{K}\left(\top_{x}\right)(z) & \leq \mathcal{K}\left(\mathcal{K}^{*}\left(\top_{x}\right)\right)(z) \\
& =\mathcal{K}\left(\bigvee_{y \in X}\left(\mathcal{K}^{*}\left(\top_{x}\right)(y) \odot \top_{y}\right)\right)(z) \\
& =\bigwedge_{y \in X}\left(\mathcal{K}^{*}\left(\top_{x}\right)(y) \rightarrow \mathcal{K}\left(\top_{y}\right)(z)\right. \\
& =\left(\bigvee_{y \in X}\left(\mathcal{K}^{*}\left(\top_{x}\right)(y) \odot \mathcal{K}^{*}\left(\top_{y}\right)(z)\right)\right)^{*}
\end{aligned}
$$

Hence $\left.\left(\mathcal{K}^{*}\left(\top_{x}\right)(y) \odot \mathcal{K}^{*}\left(\top_{y}\right)(z)\right)\right)^{*} \leq \mathcal{K}^{*}\left(\top_{x}\right)(z)$, that is, $\bigvee_{y \in X}\left(R^{*}(x, y) \odot\right.$ $\left.R^{*}(y, z)\right) \leq R^{*}(x, z)$.

$(\Leftarrow)$ Since $R^{*}$ is an $L$-fuzzy preorder, $R^{*}(x, y) \odot R^{*}(y, z) \leq R^{*}(x, z)$ iff $R^{*}(x, y) \leq R^{*}(y, z) \rightarrow R^{*}(x, z)=R(x, z) \rightarrow R(y, z)$ iff $R(x, z) \leq R^{*}(x, y) \rightarrow$ $R(y, z)$, we have

$$
\begin{aligned}
\mathcal{K}\left(\mathcal{K}^{*}(A)\right)(z) & =\bigwedge_{y \in X}\left(\mathcal{K}^{*}(A)(y) \rightarrow R(y, z)\right) \\
& =\bigwedge_{y \in X}\left(\bigvee_{x \in X}\left(A(x) \odot R^{*}(x, y)\right) \rightarrow R(y, z)\right) \\
& =\bigwedge_{y \in X} \bigwedge_{x \in X}\left(A(x) \rightarrow\left(R^{*}(x, y) \rightarrow R(y, z)\right)\right) \\
& =\bigwedge_{x \in X}\left(A(x) \rightarrow \bigwedge_{y \in X}\left(R^{*}(x, y) \rightarrow R(y, z)\right)\right) \\
& \geq \bigwedge_{x \in X}(A(x) \rightarrow R(x, z))=\mathcal{K}(A)(z) .
\end{aligned}
$$

(3) $(\Rightarrow)$ By (1), we define $R(x, y)=\mathcal{K}\left(\top_{x}\right)(y)$. Since $\mathcal{K}\left(\mathcal{K}\left(\top_{x}\right)\right)(z) \geq$ $\mathcal{K}^{*}\left(\top_{x}\right)(z)$ and $\mathcal{K}\left(\top_{x}\right)=\bigvee_{y \in X}\left(\mathcal{K}\left(\top_{x}\right)(y) \odot \top_{y}\right.$, we have

$$
\begin{aligned}
\mathcal{K}^{*}\left(\top_{x}\right)(z) & \leq \mathcal{K}\left(\mathcal{K}\left(\top_{x}\right)\right)(z) \\
& =\mathcal{K}\left(\bigvee_{y \in X}\left(\mathcal{K}\left(\top_{x}\right)(y) \odot \top_{y}\right)\right)(z) \\
& =\bigwedge_{y \in X}\left(\mathcal{K}\left(\top_{x}\right)(y) \rightarrow \mathcal{K}\left(\top_{y}\right)(z)\right. \\
& =\bigwedge_{y \in X}\left(\mathcal{K}^{*}\left(\top_{y}\right)(z) \rightarrow \mathcal{K}^{*}\left(\top_{x}\right)(y)\right.
\end{aligned}
$$


Hence $\bigvee_{z \in X} \mathcal{K}^{*}\left(\top_{x}\right)(z) \odot \mathcal{K}^{*}\left(\top_{y}\right)(z) \leq \mathcal{K}^{*}\left(\top_{x}\right)(y)$, that is, $\bigvee_{z \in X}\left(R^{*}(x, z) \odot\right.$ $\left.R^{*}(y, z)\right) \leq R^{*}(x, y)$. So, $R^{*}$ is Euclidean.

$(\Leftarrow)$ Since $R^{*}$ is Euclidean, $\left.A(x) \odot R^{*}(x, z) \odot R^{*}(y, z)\right) \leq A(x) \odot R^{*}(x, y)$ iff $A(x) \odot R^{*}(x, z) \leq R^{*}(y, z) \rightarrow A(x) \odot R^{*}(x, y)$, we have

$$
\begin{aligned}
\mathcal{K}(\mathcal{K}(A))(z) & =\bigwedge_{y \in X}(\mathcal{K}(A)(y) \rightarrow R(y, z)) \\
& =\bigwedge_{y \in X}\left(\bigwedge_{x \in X}(A(x) \rightarrow R(x, y)) \rightarrow R(y, z)\right) \\
& =\bigwedge_{y \in X}\left(R^{*}(y, z) \rightarrow\left(\bigwedge_{x \in X}(A(x) \rightarrow R(x, y))\right)^{*}\right) \\
& =\bigwedge_{y \in X}\left(R^{*}(y, z) \rightarrow \bigvee_{x \in X}\left(A(x) \odot R^{*}(x, y)\right)\right) \\
& \geq \bigvee_{x \in X}\left(A(x) \odot R^{*}(x, z)\right)=\mathcal{K}(A)(z)
\end{aligned}
$$

Theorem 7. (1) A map $\mathcal{M}: L^{X} \rightarrow L^{X}$ is an $L$-meet join approximation operator iff there exists a reflexive $L$-fuzzy relation $R \in L^{X \times X}$ such that

$$
\mathcal{M}(A)(y)=\bigvee_{x \in X}\left(A^{*}(x) \odot R(x, y)\right)
$$

(2) A map $\mathcal{K}: L^{X} \rightarrow L^{X}$ is an $L$-join meet approximation operator with $\mathcal{M}\left(\mathcal{M}^{*}(A)\right)=\mathcal{M}(A)$ iff there exists an $L$-fuzzy preorder $R \in L^{X \times X}$ such that

$$
\mathcal{M}(A)(y)=\bigvee_{x \in X}\left(A^{*}(x) \odot R(x, y)\right) .
$$

(3) A map $\mathcal{M}: L^{X} \rightarrow L^{X}$ is an $L$-meet join approximation operator with $\mathcal{M}(\mathcal{M}(A))=\mathcal{M}^{*}(A)$ iff there exists reflexive and Euclidean $L$-fuzzy relation $R \in L^{X \times X}$ such that

$$
\mathcal{M}(A)(y)=\bigvee_{x \in X}\left(A^{*}(x) \odot R(x, y)\right) .
$$
have

Proof. $\Rightarrow$ Define $R(x, y)=\mathcal{M}\left(\top_{x}^{*}\right)(y)$. Since $A=\bigwedge_{x \in X}\left(A^{*}(x) \rightarrow \top_{x}^{*}\right)$, we

$$
\begin{aligned}
\mathcal{M}(A)(y) & =\mathcal{M}\left(\bigwedge_{x \in X}\left(A(x)^{*} \rightarrow \top_{x}^{*}\right)(y)\right. \\
& =\bigvee_{x \in X}\left(A(x)^{*} \odot \mathcal{M}\left(\top_{x}^{*}\right)(y)\right) \\
& =\bigvee_{x \in X}\left(A(x)^{*} \odot R(x, y)\right) .
\end{aligned}
$$

$(2)(\Rightarrow)$ By $(1)$, we define $R(x, y)=\mathcal{M}\left(\top_{x}^{*}\right)(y)$. Since $\mathcal{M}\left(\mathcal{M}^{*}\left(\top_{x}^{*}\right)\right)(z) \leq$ $\mathcal{M}\left(\top_{x}^{*}\right)(z)$ and $\mathcal{M}^{*}\left(\top_{x}^{*}\right)=\bigwedge_{y \in X}\left(\mathcal{M}\left(\top_{x}^{*}\right)(y) \rightarrow \top_{y}^{*}\right)$, we have

$$
\begin{aligned}
& \mathcal{M}\left(\mathcal{M}^{*}\left(\top_{x}^{*}\right)\right)(z) \\
& =\mathcal{M}\left(\bigwedge_{y \in X}\left(\mathcal{M}\left(\top_{x}^{*}\right)(y) \rightarrow \top_{y}^{*}\right)\right)(z) \\
& =\bigvee_{y \in X}\left(\mathcal{M}\left(\top_{x}^{*}\right)(y) \odot \mathcal{M}\left(\top_{y}^{*}\right)(z)\right) \leq \mathcal{M}\left(\top_{x}^{*}\right)(z) \\
& \Leftrightarrow \bigvee_{z \in X}(R(x, y) \odot R(y, z)) \leq R(x, y)
\end{aligned}
$$


$(\Leftarrow)$

$$
\begin{aligned}
\mathcal{M}\left(\mathcal{M}^{*}(A)\right)(z) & =\bigvee_{y \in X}(\mathcal{M}(A)(y) \odot R(y, z)) \\
& =\bigvee_{y \in X}\left(\bigvee_{x \in X}\left(A^{*}(x) \odot R(x, y)\right) \odot R(y, z)\right) \\
& \leq \bigwedge_{x \in X}\left(A^{*}(x) \odot R(x, z)\right)=\mathcal{M}(A)(z)
\end{aligned}
$$

$(3)(\Rightarrow)$ By $(1)$, we define $R(x, y)=\mathcal{M}\left(\top_{x}^{*}\right)(y)$. Since $\mathcal{M}\left(\mathcal{M}\left(\top_{x}^{*}\right)\right)(z) \leq$ $\mathcal{M}^{*}\left(\top_{x}^{*}\right)(z)$ and $\mathcal{M}\left(\top_{x}^{*}\right)=\bigwedge_{y \in X}\left(\mathcal{M}^{*}\left(\top_{x}^{*}\right)(y) \rightarrow \top_{y}^{*}\right)$, we have

$$
\begin{aligned}
& \mathcal{M}\left(\mathcal{M}\left(\top^{*}\right)\right)(z) \\
& =\mathcal{M}\left(\bigwedge_{y \in X}\left(\mathcal{M}^{*}\left(\top_{x}^{*}\right)(y) \rightarrow \top_{y}^{*}\right)\right)(z) \\
& =\bigvee_{y \in X}\left(\mathcal{M}^{*}\left(\top_{x}^{*}\right)(y) \odot \mathcal{M}\left(\top_{y}^{*}\right)(z)\right) \leq \mathcal{M}^{*}\left(\top_{x}^{*}\right)(z) \\
& \Leftrightarrow \bigwedge_{y \in X}\left(\mathcal{M}\left(\top_{y}^{*}\right)(z) \rightarrow \mathcal{M}\left(\top_{x}^{*}\right)(y) \geq \mathcal{M}\left(\top_{x}^{*}\right)(z)\right. \\
& \Leftrightarrow \bigvee_{z \in X}\left(\mathcal{M}\left(\top_{x}^{*}\right)(z) \odot \mathcal{M}\left(\top_{y}^{*}\right)(z)\right) \leq \mathcal{M}\left(\top_{x}^{*}\right)(y) \\
& \Leftrightarrow \bigvee_{z \in X}(R(x, z) \odot R(y, z)) \leq R(x, y)
\end{aligned}
$$

$(\Leftarrow)$

$$
\begin{aligned}
\mathcal{M}(\mathcal{M}(A))(z) & =\bigvee_{y \in X}\left(\mathcal{M}^{*}(A)(y) \odot R(y, z)\right) \\
& =\bigvee_{y \in X}\left(\bigwedge_{x \in X}(R(x, y) \rightarrow A(x)) \odot R(y, z)\right) \\
& \leq \bigwedge_{x \in X}(R(x, z) \rightarrow A(x))=\mathcal{M}^{*}(A)(z)
\end{aligned}
$$

Definition 8. $[4,5]$ A subset $\tau \subset L^{X}$ is called an Alexandrov L-topology if it satisfies:

(T1) $\perp_{X}, \top_{X} \in \tau$ where $\top_{X}(x)=\top$ and $\perp_{X}(x)=\perp$ for $x \in X$.

(T2) If $A_{i} \in \tau$ for $i \in \Gamma, \bigvee_{i \in \Gamma} A_{i}, \bigwedge_{i \in \Gamma} A_{i} \in \tau$.

(T3) $\alpha \odot A \in \tau$ for all $\alpha \in L$ and $A \in \tau$.

(T4) $\alpha \rightarrow A \in \tau$ for all $\alpha \in L$ and $A \in \tau$.

Theorem 9. (1) $\tau$ is an Alexandrov L-topology on $X$ iff $\tau_{*}=\left\{A^{*} \in L^{X}\right.$ $A \in \tau\}$ is an Alexandrov L-topology on $X$.

(2) If $\mathcal{K}$ is an $L$-join meet approximation operator, then $\tau_{\mathcal{K}}=\left\{A \in L^{X} \mid\right.$ $\left.\mathcal{K}(A)=A^{*}\right\}$ is an Alexandrov L-topology on $X$.

(3) If $\mathcal{K}$ is an $L$-join meet approximation operator with $\mathcal{K}\left(\mathcal{K}^{*}(A)\right)=\mathcal{K}(A)$, then $\tau_{\mathcal{K}}=\left\{A \in L^{X} \mid \mathcal{K}(A)=A^{*}\right\}=\left\{\mathcal{K}^{*}(A) \mid A \in L^{X}\right\}$ is an Alexandrov $L$-topology on $X$.

(4) If $\mathcal{K}$ is an $L$-join meet approximation operator with $\mathcal{K}(\mathcal{K}(A))=\mathcal{K}^{*}(A)$, then $\tau_{\mathcal{K}}=\left\{\mathcal{K}(A) \mid A \in L^{X}\right\}=\left(\tau_{\mathcal{K}}\right)_{*}$ is an Alexandrov L-topology on $X$.

(5) If $\mathcal{M}$ is an $L$-meet join operator, then $\tau_{\mathcal{M}}=\left\{A \in L^{X} \mid \mathcal{M}(A)=A^{*}\right\}$ is an Alexandrov L-topology on $X$. 
(6) If $\mathcal{M}$ is an $L$-meet join approximation operator with $\mathcal{M}\left(\mathcal{M}^{*}(A)\right)=$ $\mathcal{M}(A)$, then $\tau_{\mathcal{M}}=\left\{A \in L^{X} \mid \mathcal{M}(A)=A^{*}\right\}=\left\{\mathcal{M}^{*}(A) \mid A \in L^{X}\right\}$ is an Alexandrov L-topology on $X$.

(7) If $\mathcal{M}$ is an $L$-meet join approximation operator with $\mathcal{M}(\mathcal{M}(A))=$ $\mathcal{M}^{*}(A)$, then $\tau_{\mathcal{M}}=\left\{\mathcal{M}(A) \mid A \in L^{X}\right\}=\left(\tau_{\mathcal{M}}\right)_{*}$ is an Alexandrov $L$-topology on $X$.

Proof. (1) Let $A^{*} \in \tau_{*}$ for $A \in \tau$. Since $\alpha \odot A^{*}=(\alpha \rightarrow A)^{*}$ and $\alpha \rightarrow A^{*}=$ $(\alpha \odot A)^{*}, \tau_{*}$ is an Alexandrov $L$-topology on $X$.

(2) (T1) Since $\mathcal{K}\left(\top_{X}\right) \leq \top_{X}^{*}=\perp_{X}$ and $\mathcal{K}\left(\perp_{X}\right)=\mathcal{K}(\perp \odot A)=\perp \rightarrow \mathcal{K}(A)=$ $\top_{X}, \perp_{X}=\mathcal{K}\left(\top_{X}\right)$ and $\top_{X}=\mathcal{K}\left(\perp_{X}\right)$. Then $\perp_{X}, \top_{X} \in \tau_{\mathcal{K}}$.

(T2) For $A_{i} \in \tau_{\mathcal{K}}$ for each $i \in \Gamma$, by (K3),

$$
\mathcal{K}\left(\bigvee_{i \in \Gamma} A_{i}\right)=\bigwedge_{i \in \Gamma} \mathcal{K}\left(A_{i}\right)=\bigwedge_{i \in \Gamma} A_{i}^{*}=\left(\bigvee_{i \in \Gamma} A_{i}\right)^{*}
$$

Thus $\bigvee_{i \in \Gamma} A_{i} \in \tau_{\mathcal{K}}$. Since $\mathcal{K}$ is a deceasing map for (K3), we have

$$
\bigvee_{i \in \Gamma} A_{i}^{*}=\bigvee_{i \in \Gamma} \mathcal{K}\left(A_{i}\right) \leq \mathcal{K}\left(\bigwedge_{i \in \Gamma} A_{i}\right) \leq\left(\bigwedge_{i \in \Gamma} A_{i}\right)^{*},
$$

Thus, $\bigwedge_{i \in \Gamma} A_{i} \in \tau_{\mathcal{K}}$.

(T3) For $A \in \tau_{\mathcal{K}}$, by $(\mathrm{K} 2), \alpha \odot A \in \tau_{\mathcal{K}}$.

(T4) For $A \in \tau_{\mathcal{K}}$, by Lemma $5(1), \mathcal{K}(\alpha \rightarrow A) \geq \alpha \odot \mathcal{K}(A)=\alpha \odot A^{*}=(\alpha \rightarrow$ $A)^{*}$. Then $\alpha \rightarrow A \in \tau_{\mathcal{K}}$.

(3) Put $P=\left\{\mathcal{K}^{*}(A) \mid A \in L^{X}\right\}$. Let $\mathcal{K}^{*}(A) \in P$. Since $\mathcal{K}\left(\mathcal{K}^{*}(A)\right)=\mathcal{K}(A)$, then $\mathcal{K}^{*}(A) \in \tau_{\mathcal{K}}$. Let $A \in \tau_{\mathcal{K}}$. Then $A=\mathcal{K}^{*}(A) \in P$.

(4) Let $A \in \tau_{\mathcal{K}}$. Then $A^{*}=\mathcal{K}(A)$. Since $\mathcal{K}(\mathcal{K}(A))=\mathcal{K}^{*}(A)$, then $\mathcal{K}\left(A^{*}\right)=$ $A$. So $A^{*} \in\left(\tau_{\mathcal{K}}\right)_{*}$. Let $A \in\left(\tau_{\mathcal{K}}\right)_{*}$. Then $A=\mathcal{K}\left(A^{*}\right)$. Since $\mathcal{K}\left(\mathcal{K}\left(A^{*}\right)\right)=$ $\mathcal{K}^{*}\left(A^{*}\right)$, then $\mathcal{K}(A)=A^{*}$. So $A \in \tau_{\mathcal{K}}$. Hence $\tau_{\mathcal{K}}=\left(\tau_{\mathcal{K}}\right)_{*}$. Put $Q=\{\mathcal{K}(A) \mid A \in$ $L^{X}$. Let $\mathcal{K}(A) \in Q$. Since $\mathcal{K}(\mathcal{K}(A))=\mathcal{K}^{*}(A)$, then $\mathcal{K}(A) \in \tau_{\mathcal{K}}$. Let $A \in \tau_{\mathcal{K}}$. Then $A^{*}=\mathcal{K}(A) \in\left(\tau_{\mathcal{K}}\right)_{*}=\tau_{\mathcal{K}}$. Hence $Q=\tau_{\mathcal{K}}$.

(5) (T1) Since $\perp_{X}^{*} \leq \mathcal{M}\left(\perp_{X}\right)$ and $\mathcal{M}\left(\top_{X}\right)=\mathcal{M}\left(\perp_{X} \rightarrow A\right)=\perp_{X} \odot$ $\mathcal{M}(A)=\perp_{X}, \top_{X}=\mathcal{M}\left(\perp_{X}\right)$ and $\perp_{X}=\mathcal{M}\left(\top_{X}\right)$. Then $\perp_{X}, \top_{X} \in \tau_{\mathcal{M}}$.

(T2) For $A_{i} \in \tau_{\mathcal{M}}$ for each $i \in \Gamma$, by (M3),

$$
\mathcal{M}\left(\bigwedge_{i \in \Gamma} A_{i}\right)=\bigvee_{i \in \Gamma} \mathcal{M}\left(A_{i}\right)=\bigvee_{i \in \Gamma} A_{i}^{*}=\left(\bigwedge_{i \in \Gamma} A_{i}\right)^{*} .
$$

Thus, $\bigwedge_{i \in \Gamma} A_{i} \in \tau_{\mathcal{M}}$. Since $\mathcal{M}$ is deceasing map for (M3), we have

$$
\left(\bigvee_{i \in \Gamma} A_{i}\right)^{*} \leq \mathcal{M}\left(\bigvee_{i \in \Gamma} A_{i}\right) \leq \bigwedge_{i \in \Gamma} \mathcal{M}\left(A_{i}\right)=\left(\bigvee_{i \in \Gamma} A_{i}\right)^{*},
$$


Thus, $\bigvee_{i \in \Gamma} A_{i} \in \tau_{\mathcal{M}}$.

(T3) For $A \in \tau_{\mathcal{M}}$, by Lemma $5(2),(\alpha \odot A)^{*} \leq \mathcal{M}(\alpha \odot A) \leq \alpha \rightarrow \mathcal{M}(A)=$ $\alpha \rightarrow A^{*}$. Then $\alpha \odot A \in \tau_{\mathcal{M}}$.

(T4) For $A \in \tau_{\mathcal{M}}$, by (M2), $\mathcal{M}(\alpha \rightarrow A)=\alpha \odot \mathcal{M}(A)=\alpha \odot A^{*}=(\alpha \rightarrow A)^{*}$. Then $\alpha \rightarrow A \in \tau_{\mathcal{M}}$.

(6) and (7) are similarly proved as (3) and (4), respectively.

Theorem 10. Let $R \in L^{X \times X}$ be an $L$-fuzzy relation. Define operators as follows

$$
\begin{aligned}
\mathcal{K}_{R^{*}}(A)(y) & =\bigwedge_{x \in X}\left(A(x) \rightarrow R^{*}(x, y)\right) \\
\mathcal{K}_{R^{-1 *}}(A)(y) & =\bigwedge_{x \in X}\left(A(x) \rightarrow R^{-1 *}(x, y)\right) \\
\mathcal{M}_{R}(A)(y) & =\bigvee_{x \in X}\left(A^{*}(x) \odot R(x, y)\right) \\
\mathcal{M}_{R^{-1}}(A)(y) & =\bigvee_{x \in X}\left(A^{*}(x) \odot R^{-1}(x, y)\right)
\end{aligned}
$$

Then the following properties hold.

(1) If $R$ is reflexive, then $\tau_{\mathcal{K}_{\mathrm{R}}}=\tau_{\left(\mathcal{K}_{\mathrm{R}-1 *}\right)_{*}}=\tau_{\mathcal{M}_{\mathrm{R}-1}}=\tau_{\left(\mathcal{M}_{\mathrm{R}}\right)_{*}}$.

(2) If $R$ is an $L$-fuzzy preorder, then

$$
\begin{aligned}
\tau_{\mathcal{K}_{\mathrm{R} *}} & =\left\{\bigvee_{x \in X}(A(x) \odot R(x,-)) \mid A \in L^{X}\right\} \\
\tau_{\mathcal{K}_{\mathrm{R}-1 *}} & =\left\{\bigvee_{x \in X}(A(x) \odot R(-, x)) \mid A \in L^{X}\right\} \\
\tau_{\mathcal{M}_{\mathrm{R}}} & =\left\{\bigwedge_{x \in X}(R(x,-) \rightarrow A(x)) \mid A \in L^{X}\right\} \\
\tau_{\mathcal{M}_{\mathrm{R}-1}} & =\left\{\bigwedge_{x \in X}(R(-, x) \rightarrow A(x)) \mid A \in L^{X}\right\} .
\end{aligned}
$$

(3) If $R$ is reflexive and Euclidean, then $R$ is symmetric, $R$ is an $L$-fuzzy preorder and $\tau_{\mathcal{K}_{\mathrm{R}} *}=\tau_{\mathcal{K}_{\mathrm{R}-1 *}}=\tau_{\left(\mathcal{K}_{\mathrm{R}-1 *}\right)_{*}}=\tau_{\mathcal{M}_{\mathrm{R}-1}}=\tau_{\mathcal{M}_{\mathrm{R}}}=\tau_{\left(\mathcal{M}_{\mathrm{R}}\right)_{*}}$ such that

$$
\begin{aligned}
\tau_{\mathcal{K}_{\mathrm{R} *}} & =\left\{\bigwedge_{x \in X}\left(A(x) \rightarrow R^{*}(x,-)\right) \mid A \in L^{X}\right\} \\
\tau_{\mathcal{K}_{\mathrm{R}-1 *}} & =\left\{\bigwedge_{x \in X}\left(A(x) \rightarrow R^{*}(-, x)\right) \mid A \in L^{X}\right\} \\
\tau_{\mathcal{M}_{\mathrm{R}}} & =\left\{\bigvee_{x \in X}\left(R(x,-) \odot A^{*}(x)\right) \mid A \in L^{X}\right\} \\
\tau_{\mathcal{M}_{\mathrm{R}-1}} & =\left\{\bigvee_{x \in X}\left(R(-, x) \odot A^{*}(x)\right) \mid A \in L^{X}\right\} .
\end{aligned}
$$

Proof. (1) We only show that

$$
\begin{aligned}
& \mathcal{K}_{R^{*}}(A)=A^{*} \text { iff } \mathcal{K}_{R^{-1 *}}\left(A^{*}\right)=A \\
& \text { iff } \mathcal{M}_{R}\left(A^{*}\right)=A \text { iff } \mathcal{M}_{R^{-1}}(A)=A^{*} .
\end{aligned}
$$

First, $\mathcal{K}_{R^{*}}(A)=A^{*}$ iff $\mathcal{K}_{R^{-1 *}}\left(A^{*}\right)=A$.

Since $\mathcal{K}_{R^{*}}(A)(y)=\bigwedge_{x \in X}\left(A(x) \rightarrow R^{*}(x, y)\right) \geq A^{*}(y)$, then

$$
A(x) \leq \bigwedge_{y \in X} A^{*}(y) \rightarrow R^{*}(x, y),
$$


iff $A(x) \leq \mathcal{K}_{R^{-1 *}}\left(A^{*}\right)(x)$. Hence $\mathcal{K}_{R^{-1 *}}\left(A^{*}\right)=A$. Conversely, it is similarly proved.

Second, $\mathcal{K}_{R^{*}}(A)=A^{*}$ iff $\mathcal{M}_{R}\left(A^{*}\right)=A$.

Since $\mathcal{K}_{R^{*}}(A)(y)=\bigwedge_{x \in X}\left(A(x) \rightarrow R^{*}(x, y) \geq A^{*}(y)\right.$, then $\left(\bigwedge_{x \in X}(A(x) \rightarrow\right.$ $\left.R^{*}(x, y)\right)^{*}=\bigvee_{x \in X}(A(x) \odot R(x, y))=\mathcal{M}_{R}\left(A^{*}\right)(y) \leq A(y)$. Thus $\mathcal{M}_{R}\left(A^{*}\right)=A$. Conversely, it is similarly proved.

Finally, by a second method, we have $\mathcal{K}_{R^{-1 *}}\left(A^{*}\right)=A$ iff $\mathcal{M}_{R^{-1}}(A)=A^{*}$.

(2) Since $R$ and $R^{-1}$ are $L$-fuzzy preorders, by Theorems $6(2)$ and $7(2)$, then

$$
\begin{aligned}
\mathcal{K}_{R^{*}}\left(\mathcal{K}_{R^{*}}^{*}(A)\right)=\mathcal{K}_{R^{*}}(A), \mathcal{K}_{R^{-1 *}}\left(\mathcal{K}_{R^{-1 *}}^{*}(A)\right)=\mathcal{K}_{R^{-1 *}}(A) \\
\mathcal{M}_{R}\left(\mathcal{M}_{R}^{*}(A)\right)=\mathcal{M}_{R}(A), \mathcal{M}_{R^{-1}}\left(\mathcal{M}_{R^{-1}}^{*}(A)\right)=\mathcal{M}_{R^{-1}}(A), \\
\tau_{\mathcal{K}_{\mathrm{R}}} \quad=\left\{\mathcal{K}_{R^{*}}^{*}(A)=\bigvee_{x \in X}(A(x) \odot R(x,-)) \mid A \in L^{X}\right\} \\
\tau_{\mathcal{K}_{\mathrm{R}-1 *}}=\left\{\mathcal{K}_{R^{-1 *}}(A)=\bigvee_{x \in X}(A(x) \odot R(-, x)) \mid A \in L^{X}\right\} \\
\tau_{\mathcal{M}_{\mathrm{R}}} \quad=\left\{\mathcal{M}_{R}^{*}(A)=\bigwedge_{x \in X}(R(x,-) \rightarrow A(x)) \mid A \in L^{X}\right\} \\
\tau_{\mathcal{M}_{\mathrm{R}-1}}=\left\{\mathcal{M}_{R-1}^{*}(A)=\bigwedge_{x \in X}(R(-, x) \rightarrow A(x)) \mid A \in L^{X}\right\} .
\end{aligned}
$$

(2) Since $R$ and $R^{-1}$ are reflexive and Euclidean $L$-fuzzy preorder, by Theorems $6(3)$ and $7(3)$,

$$
\begin{gathered}
\mathcal{K}_{R^{*}}\left(\mathcal{K}_{R^{*}}(A)\right)=\mathcal{K}_{R^{*}}^{*}(A), \mathcal{K}_{R^{-1 *}}\left(\mathcal{K}_{R^{-1 *}}(A)\right)=\mathcal{K}_{R^{-1 *}}^{*}(A) \\
\mathcal{M}_{R}\left(\mathcal{M}_{R}(A)\right)=\mathcal{M}_{R}^{*}(A), \mathcal{M}_{R^{-1}}\left(\mathcal{M}_{R^{-1}}(A)\right)=\mathcal{M}_{R^{-1}}^{*}(A), \\
\tau_{\mathcal{K}_{\mathrm{R} *}}=\left\{\mathcal{K}_{R^{*}}(A)=\bigwedge_{x \in X}\left(A(x) \rightarrow R^{*}(x,-)\right) \mid A \in L^{X}\right\} \\
\tau_{\mathcal{K}_{\mathrm{R}}-1 *}=\left\{\mathcal{K}_{R^{-1 *}}(A)=\bigwedge_{x \in X}\left(A(x) \rightarrow R^{*}(-, x)\right) \mid A \in L^{X}\right\} \\
\tau_{\mathcal{M}_{\mathrm{R}}}=\left\{\mathcal{M}_{R}(A)=\bigvee_{x \in X}\left(R(x,-) \odot A^{*}(x)\right) \mid A \in L^{X}\right\} \\
\tau_{\mathcal{M}_{\mathrm{R}-1}}=\left\{\mathcal{M}_{R-1}(A)=\bigvee_{x \in X}\left(R(-, x) \odot A^{*}(x)\right) \mid A \in L^{X}\right\} .
\end{gathered}
$$

Example 11. Let $\left(L=[0,1], \odot, \rightarrow,{ }^{*}\right)$ be a complete residuated lattice with the law of double negation defined by

$$
x \odot y=(x+y-1) \vee 0, x \rightarrow y=(1-x+y) \wedge 1, x^{*}=1-x .
$$

Let $X=\{a, b, c\}$ and we define $R \in L^{X \times X}$ as follows

$$
R=\left(\begin{array}{ccc}
1 & 0.7 & 0.3 \\
0.8 & 1 & 0.4 \\
0.9 & 0.6 & 1
\end{array}\right)
$$


(1) Since $R$ is a reflexive $L$-fuzzy relation, by Theorem $6(1), \mathcal{K}_{R^{*}}$ is an $L$-join meet approximation operator. Moreover, by Theorem $7(1), \mathcal{M}_{R}$ is an $L$-join meet approximation operator.

(2) Since $R$ is an $L$-fuzzy preorder on $X$, by Theorem $10(1)$, we have

$$
\tau_{\mathcal{K}_{\mathrm{R}} *}=\tau_{\left(\mathcal{K}_{\mathrm{R}-1 *}\right)_{*}}=\tau_{\mathcal{M}_{\mathrm{R}-1}}=\tau_{\left(\mathcal{M}_{\mathrm{R}-1}\right)_{*}} \cdot
$$

(3) For $A(a)=0.7, A(b)=0.5, A(c)=0.5$, since $R$ is an $L$-fuzzy preorder on $X$, by Theorem $7(2)$,

$$
\begin{gathered}
\mathcal{K}_{R^{*}}(A)=(0.3,0.5,0.5)=\mathcal{K}_{R^{*}}\left(\mathcal{K}_{R^{*}}^{*}(A)\right) . \\
\mathcal{K}_{R^{*}}^{*}(A)=(0.7,0.5,0.5) \in \tau_{\mathcal{K}_{\mathrm{R}^{*}}} .
\end{gathered}
$$

Since $0.9=\bigvee_{x \in X} R(a, x) \odot R(c, x) \not \subset R(a, c)=0.3, R$ is not Euclidean. By Theorem 6(3), we have

$$
\begin{gathered}
\mathcal{K}_{R^{*}}^{*}(A)=(0.7,0.5,0.5) \neq \mathcal{K}_{R^{*}}\left(\mathcal{K}_{R^{*}}(A)\right)=(0.6,0.5,0.5) . \\
\mathcal{K}_{R^{*}}(A)=(0.3,0.5,0.5) \notin \tau_{\mathcal{K}_{\mathrm{R}^{*}}} .
\end{gathered}
$$

For $B(a)=0.4, B(b)=0.5, B(c)=0.6$, since $R$ is an $L$-fuzzy preorder on $X$, by Theorem $7(2)$,

$$
\begin{gathered}
\mathcal{M}_{R}(B)=(0.6,0.5,0.4)=\mathcal{M}_{R}\left(\mathcal{M}_{R}^{*}(B)\right) . \\
\mathcal{M}_{R}^{*}(B)=(0.4,0.5,0.6) \in \tau_{\mathcal{M}_{\mathrm{R}}} .
\end{gathered}
$$

Since $0.9=\bigvee_{x \in X} R(a, x) \odot R(c, x) \not \subset R(a, c)=0.3, R$ is not Euclidean. By Theorem 6(3),

$$
\begin{gathered}
\mathcal{M}_{R}^{*}(B)=(0.4,0.5,0.6) \neq \mathcal{M}_{R}\left(\mathcal{M}_{R}(B)\right)=(0.5,0.5,0.6) . \\
\mathcal{M}_{R}(B)=(0.6,0.5,0.4) \notin \tau_{\mathcal{M}_{\mathrm{R}}} .
\end{gathered}
$$
$R^{(2)}$ as

(4) Put $R^{(2)}(y, z)=\bigvee_{x \in X}(R(y, x) \odot R(z, x))$, we obtain an $L$-fuzzy relation

$$
R^{(2)}=\left(\begin{array}{ccc}
1 & 0.8 & 0.9 \\
0.8 & 1 & 0.7 \\
0.9 & 0.7 & 1
\end{array}\right) .
$$

Since $R^{(2)}$ is Euclidean and $R^{(2)}(x, y)=R^{(2)}(y, x)$ for all $x, y \in X$, then $R^{(2)}$ is an $L$-fuzzy preorder. By Theorems 6 and 7 , for $C \in L^{X}$, we obtain

$$
\mathcal{K}_{R^{(2) *}}(C)=\mathcal{K}_{R^{(2)}}\left(\mathcal{K}_{R^{(2) *}}^{*}(C)\right)
$$




$$
\begin{aligned}
& \mathcal{K}_{R^{(2) *}}^{*}(C)=\mathcal{K}_{R^{(2) *}}\left(\mathcal{K}_{R^{(2) *}}(C)\right), \\
& \mathcal{M}_{R^{(2)}}^{*}(C)=\mathcal{M}_{R^{(2)}}\left(\mathcal{M}_{R^{(2)}}(C)\right), \\
& \mathcal{M}_{R^{(2)}}(C)=\mathcal{M}_{R^{(2)}}\left(\mathcal{M}_{R^{(2)}}^{*}(C)\right) .
\end{aligned}
$$

By Theorem 10(3), we have

$$
\tau_{\mathcal{K}_{\mathrm{R}(2) *}}=\tau_{\left(\mathcal{K}_{\mathrm{R}(2)-1 *}\right)_{*}}=\tau_{\left(\mathcal{K}_{\mathrm{R}(2) *}\right)_{*}}=\tau_{\mathcal{M}_{\mathrm{R}(2)}}=\tau_{\mathcal{M}_{\mathrm{R}(2)-1}}=\tau_{\left(\mathcal{M}_{\mathrm{R}(2)}\right)_{*}} .
$$

(5) Since $0.9=\bigvee_{x \in X} R(x, a) \odot R(x, c) \not \leq R(a, c)=0.3$, for $A(a)=0.5, A(b)=$ $0.6, A(c)=0.7$, we have

$$
\mathcal{K}_{R^{-1 *}}^{*}(A)=(0.5,0.6,0.7) \neq \mathcal{K}_{R^{-1 *}}\left(\mathcal{K}_{R^{-1 *}}(A)\right)=(0.5,0.6,0.6) .
$$

Moreover, since $R^{-1}$ is an $L$-fuzzy preorder, by Theorem 9 ,

$$
\mathcal{K}_{R^{-1 *}}^{*}(A)=(0.5,0.6,0.7) \in \tau_{\mathcal{K}_{\mathrm{R}-1 *}}, \mathcal{K}_{R^{-1 *}}(A)=(0.5,0.4,0.3) \notin \tau_{\mathcal{K}_{\mathrm{R}-1 *}} .
$$

(6) For $A(a)=0.5, A(b)=0.1, A(c)=0.2$, we have

$$
\mathcal{M}_{R^{-1}}^{*}(A)=(0.4,0.1,0.2) \neq \mathcal{M}_{R^{-1}}\left(\mathcal{M}_{R^{-1}}(A)\right)=(0.4,0.2,0.3) .
$$

Moreover, since $R^{-1}$ is an $L$-fuzzy preorder, by Theorem 9 , we have

$$
\mathcal{M}_{R^{-1}}^{*}(A)=(0.4,0.1,0.2) \in \tau_{\mathcal{M}_{\mathrm{R}-1}}, \mathcal{M}_{R^{-1}}(A)=(0.6,0.9,0.8) \notin \tau_{\mathcal{M}_{\mathrm{R}-1}} .
$$

(7) Put $R^{[2]}(y, z)=\bigvee_{x \in X} R(x, y) \odot R(x, z)$, we obtain an $L$-fuzzy relation $R^{[2]}, R^{[3]}$ as

$$
R^{[2]}=\left(\begin{array}{ccc}
1 & 0.8 & 0.9 \\
0.8 & 1 & 0.6 \\
0.9 & 0.6 & 1
\end{array}\right) \quad R^{[3]}=\left(\begin{array}{ccc}
1 & 0.8 & 0.9 \\
0.8 & 1 & 0.7 \\
0.9 & 0.7 & 1
\end{array}\right) .
$$

Since $\mathcal{M}_{\left(R^{[2]}\right)^{-1}}\left(\mathcal{M}_{\left(R^{[2]}\right)^{-1}}\left(\top_{x}^{*}\right)\right)(z) \leq \mathcal{M}_{\left(R^{[2]}\right)^{-1}}^{*}\left(\top_{x}^{*}\right)(z)$ and $\mathcal{M}_{\left(R^{[2]}\right)^{-1}}\left(T_{x}^{*}\right)=$ $\bigwedge_{y \in X}\left(\mathcal{M}_{\left(R^{[2]}\right)^{-1}}^{*}\left(\top_{x}^{*}\right)(y) \rightarrow \top_{y}^{*}\right)$, we have $\mathcal{M}_{\left(R^{[2]}\right)^{-1}}\left(\top_{c}^{*}\right) \notin \tau_{\left.\mathcal{M}_{(\mathrm{R}}^{[2]}\right)_{-1}}$ from:

$$
\begin{aligned}
& 0.7=\bigvee_{x \in X} R^{[2]}(x, b) \odot R^{[2]}(x, c) \not \leq R^{[2]}(b, c)=0.6 \\
& \Leftrightarrow \bigvee_{x \in X}\left(\mathcal{M}_{\left(R^{[2]}\right)^{-1}}\left(\mathrm{~T}_{b}^{*}\right)(x) \odot \mathcal{M}_{\left(R^{[2]}\right)^{-1}}\left(\mathrm{~T}_{c}^{*}\right)(x)\right) \not \leq \mathcal{M}_{\left(R^{[2]}\right)^{-1}}\left(\mathrm{~T}_{c}^{*}\right)(b) \\
& \Leftrightarrow \bigwedge_{y \in X}\left(\mathcal{M}_{\left(R^{[2]}\right)^{-1}}\left(T_{b}^{*}\right)(x) \rightarrow \mathcal{M}_{\left(R^{[2]}\right)^{-1}}\left(T_{c}^{*}\right)(b) \not \mathcal{M}_{\left(R^{[2]}\right)^{-1}}\left(T_{c}^{*}\right)(x)\right. \\
& \Leftrightarrow \bigvee_{b \in X}\left(\mathcal{M}_{\left(R^{[2]}\right)^{-1}}\left(T_{b}^{*}\right)(x) \odot \mathcal{M}_{\left(R^{[2]}\right)^{-1}}^{*}\left(T_{c}^{*}\right)(b) \not \leq \mathcal{M}_{\left(R^{[2]}\right)^{-1}}^{*}\left(T_{c}^{*}\right)(x)\right. \\
& \Leftrightarrow \mathcal{M}_{\left(R^{[2]}\right)^{-1}}\left(\bigwedge_{b \in X} \mathcal{M}_{\left(R^{[2]}\right)^{-1}}^{*}\left(T_{c}^{*}\right)(b) \rightarrow \top_{b}^{*}\right)(x) \not \leq \mathcal{M}_{\left(R^{[2]}\right)^{-1}}^{*}\left(\top_{c}^{*}\right)(x) \\
& \Leftrightarrow \mathcal{M}_{\left(R^{[2]}\right)^{-1}}\left(\mathcal{M}_{\left(R^{[2]}\right)^{-1}}\left(\top_{c}^{*}\right)\right)(x) \not \leq \mathcal{M}_{\left(R^{[2]}\right)^{-1}}^{*}\left(\top_{c}^{*}\right)(x)
\end{aligned}
$$


Since

$$
\begin{gathered}
\mathcal{M}_{\left(R^{[2]}\right)^{-1}}\left(\mathcal{M}_{\left(R^{[2]}\right)^{-1}}^{*}\left(1_{c}^{*}\right)\right)(b)=\bigvee_{a \in X} R^{[2]}(c, a) \odot R^{[2]}(a, b) \\
\left.\not \leq R^{[2]}(c, b)=0.6=\mathcal{M}_{\left(R^{[2]}\right)^{-1}}\left(1_{c}^{*}\right)\right)(b), \\
\mathcal{M}_{\left(R^{[2]}\right)^{-1}}^{*}\left(1_{c}^{*}\right) \notin \tau_{\left.\mathcal{M}_{(\mathrm{R}}[2]\right)^{-1}}
\end{gathered}
$$

(8) Since $R^{[3]}$ is Euclidean and $R^{[3]}(x, y)=R^{[3]}(y, x)$ for all $x, y \in X$, then $R^{[3]}$ is an $L$-fuzzy preorder. By Theorems 6 and 7 , for $C \in L^{X}$, we obtain

$$
\begin{aligned}
& \mathcal{K}_{R^{[3] *}}(C)=\mathcal{K}_{R^{[3]}}\left(\mathcal{K}_{R^{[3] *}}^{*}(C)\right), \\
& \mathcal{K}_{R^{[3] *}}^{*}(C)=\mathcal{K}_{R^{[3] *}}\left(\mathcal{K}_{R^{[3] *}}(C)\right), \\
& \mathcal{M}_{R^{[3]}}^{*}(C)=\mathcal{M}_{R^{[3]}}\left(\mathcal{M}_{R^{[3]}}(C)\right), \\
& \mathcal{M}_{R^{[3]}}(C)=\mathcal{M}_{R^{[3]}}\left(\mathcal{M}_{R^{[3]}}^{*}(C)\right) .
\end{aligned}
$$

By Theorem 10(3), we have

$$
\tau_{\mathcal{K}_{\mathrm{R}[3] *}}=\tau_{\left(\mathcal{K}_{\mathrm{R}[3]-1 *}\right)_{*}}=\tau_{\left(\mathcal{K}_{\mathrm{R}[3] *}\right)_{*}}=\tau_{\mathcal{M}_{\mathrm{R}[3]}}=\tau_{\mathcal{M}_{\mathrm{R}[3]-1}}=\tau_{\left(\mathcal{M}_{\mathrm{R}[3]}\right)_{*}} \cdot
$$

\section{References}

[1] R. Bělohlávek,Fuzzy Relational Systems, Kluwer Academic Publishers, New York , (2002).

[2] P. Hájek, Metamathematices of Fuzzy Logic, Kluwer Academic Publishers, Dordrecht (1998).

[3] Y.C. Kim, L-approximatins and join preserving maps, J. Math. Comput. Sci., 3 (5)(2013), 1193-1210.

[4] Y.C. Kim, Alexandrov L-topologies, to appear International Journal of Pure and Applied Mathematics.

[5] H. Lai, D. Zhang, Fuzzy preorder and fuzzy topology, Fuzzy Sets and Systems, 157 (2006), 1865-1885, doi: 10.1016/j.fss.2006.02.013.

[6] H. Lai, D. Zhang, Concept lattices of fuzzy contexts: Formal concept analysis vs. rough set theory, Int. J. Approx. Reasoning, 50 (2009), 695-707, doi: $10.1016 /$ j.ijar.2008.12.002.

[7] Z. Pawlak, Rough sets, Int. J. Comput. Inf. Sci., 11 (1982), 341-356. 
[8] Z. Pawlak, Rough probability, Bull. Pol. Acad. Sci. Math., 32(1984), 607615.

[9] A. M. Radzikowska, E.E. Kerre, A comparative study of fuzy rough sets, Fuzzy Sets and Systems, 126(2002), 137-155, doi: 10.1016/so1650114(01)00032-x.

[10] Y.H. She, G.J. Wang, An axiomatic approach of fuzzy rough sets based on residuated lattices, Computers and Mathematics with Applications, $\mathbf{5 8}$ (2009), 189-201, doi: 10.1016/j.camwa.2009.03.100.

[11] Zhen Ming Ma, Bao Qing Hu, Topological and lattice structures of Lfuzzy rough set determined by lower and upper sets, Information Sciences, 218(2013), 194-204, doi: 10.1016/j.ins.2012.06.029. 
